\title{
A Mean quantization based synchronous digital audio watermarking method on wavelet domain
}

\author{
Li Lijuan \\ Department of Information Engineering, Shijiazhuang Vocational Technology Institute, 050081 \\ Shijiazhuang, China \\ e-mail: lilj@sjzpt.edu.cn
}

Keywords: audio watermarking, wavelet transform, mean quantization, synchronization signals, robustness

\begin{abstract}
In this paper, a mean quantization based synchronous digital audio watermarking method on wavelet domain is proposed, which is a blind detection algorithm to detect without the original audio signal. It introduces coefficient of mean quantization algorithm, and averagely quantize low-frequency coefficients for watermarking after wavelet decomposition to audio signal. It also bring the concept of embedded synchroniztion signal to determine the position of watermark for resisting the shift, cutting and other attacks. The results of experiments validate the robustness and resistance to attack of this method.
\end{abstract}

\section{INTRODUCTION}

With the rapid development of network technique and multi-media technique, the Internet has become an important way to disseminate knowledge and information. However, the followed issues on piracy and copyright disputes become pressing problems to address. Digital watermark technology is now recognized as the solution is an effective solution to copyright issues, which can declare the ownership of digital products to identify the buyer or provide relevant information and content.

Digital Audio Watermarking algorithm is an essential part of audio watermarking technology. The most crucial properties of digital audio watermark are the non-auditory and robustness. In the common digital audio watermarking technology, because of the need for copyright protection of digital music products, transform domain audio watermarking algorithm has made great progress. In this algorithm, the literature ${ }^{[1]}$ presented a wavelet transform of the audio watermarking algorithm, which hided watermark ininvisibility and robustness.

The paper ${ }^{[2]}$ proposed a wavelet domain quantization based digital audio watermarking algorithm which extracted watermark without the original digital audio signal using modulated signals embedded by quantizing the discrete wavelet transform coefficients. The paper ${ }^{[3]}$ proposed a mean quantization digital audio watermarking in wavelet domain algorithm which played better robust to mp3, quantization, re-sampling, low pass filtering and noise and other attacks.

The paper ${ }^{[4]}$ is proposed based on auditory masking effect and the wavelet transform of the audio watermarking algorithm, which has a strong hidden nature of the watermark. The paper ${ }^{[5]}$ proposed a synchronization of digital audio watermarking algorithm, which located the embedding position of the synchronous signal in order to improve the accuracy of extracting the watermark, and added the segment information into the watermark by redundant coding. It can enhance the error correction capability.

\section{Mean Quantization Principle}

Assuming that the set of $K$ wavelet coefficients as $\left\{x_{0}, \ldots, x_{K-1}\right\}$, its mean value is $\bar{x}=\frac{1}{K} \sum_{i=0}^{K-1} x_{i}$

.Assuming in the mean $\bar{x}$, the error caused by a embedded watermark bit during the 
use of quantitative methods is $\Delta$, the mean of $\mathrm{K}$ signals after a embedded watermark bit $w_{i}$ is $\bar{x}^{*}=\bar{x}+\Delta$, It can be seen that the set of $\mathrm{K}$ wavelet coefficients of all individual coefficients will be modified accordingly, that is $x_{i}^{*}=x_{i}+\Delta, \quad i=0, \ldots, K-1$

In which $x_{i}^{*}$ is one of the modified coefficients.Suppose embedded watermark signal suffers a certain attack in the transmission process , the error generated by the attack on each signal is $\delta_{i}$,one of the $\mathrm{K}$ wavelet coefficients after the attack is $\hat{x}_{i}={ }^{*} x_{i}+\delta_{i}, i=0, \ldots, K-1$, and the mean value of K wavelet coefficients is $\overline{\hat{x}}_{i}=\frac{1}{K} \sum_{i=1}^{K-1} \hat{x}_{i}=\overline{x^{*}}+\overline{\delta_{i}}$. Assume $\delta_{i} \sim N\left(0, \sigma^{2}\right)$, which means $\delta_{i}$ obeys Gaussian distribution with mean 0 and variance $\sigma^{2}$,the average of $\mathrm{K}$ random variables obey Gaussian distribution still obeys Gaussian distribution, but the variance is reduced by $\mathrm{K}$ times, that is $\bar{\delta} \sim N\left(0, \frac{\sigma^{2}}{K}\right)$. Therefore, compared to quantitative methods with a single factor, Mean quantization can reduce the variance caused by the attack and coefficient changes in the and improve robustness.Fig. 1 shows the statistical distribution of wavelet coefficients changes.

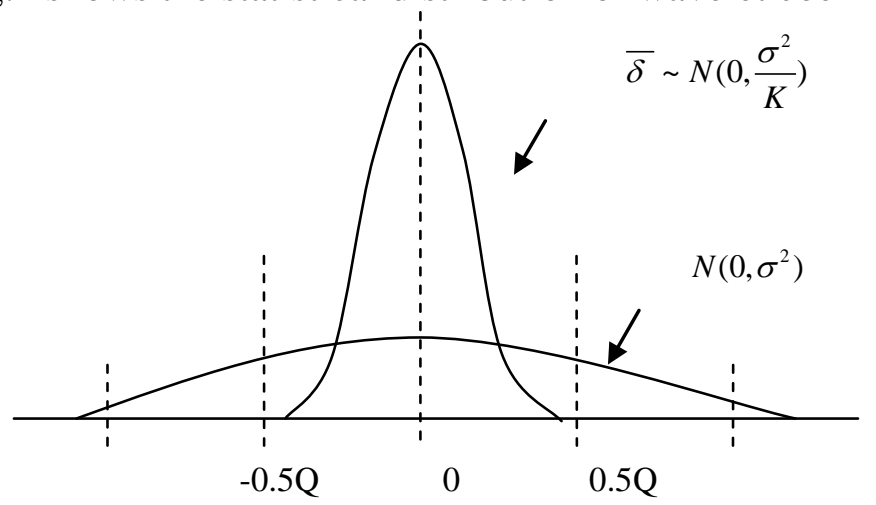

Figure 1. Gaussian distribution with different variances

\section{THE SELECTION AND DETERMINATION OF THE SYNCHRONIZATION SIGNAL}

In this paper , a m-sequence is used as the synchronous signal, first transform the m-sequence $\left\{c_{n}\right\}\left(c_{n}=1,0\right)$ into $\left\{c_{n}^{\prime}\right\}\left(c_{n}^{\prime}=1,-1\right)$, that is $c_{n}^{\prime}=2 c_{n}-1$.

Assume that $\left\{a_{n}\right\}$ and $\left\{b_{n}\right\}\left(a_{n}, b_{n}=1,-1\right)$ are two m-sequences with the same period $\mathrm{T}$, so their autocorrelation coefficient is defined as

$$
\rho_{a, b}(\tau)=\frac{1}{T} \sum_{n=1}^{T} a_{n} b_{n}-\tau
$$

The autocorrelation coefficient of m-sequence $\left\{a_{n}\right\}$ is defined as

$$
\rho_{a}(\tau)=\frac{1}{T} \sum_{n=1}^{T} a_{n} a_{n}-\tau
$$

The autocorrelation coefficient of m-sequence has the following properties:

$$
\rho_{a}(\tau)=\left\{\begin{array}{c}
1, \quad \tau=0 \\
-1 / T, \quad \tau \neq 0
\end{array}\right.
$$

Let $\left\{a_{n}\right\}$ be the original m-sequence used as a synchronization signal, $\left\{b_{n}\right\}$ is a sequence to be tested, if $\rho_{a, b}(0) \geq n / T$, then determine that $\left\{b_{n}\right\}$ is a synchronization signal, in which $\mathrm{n}$ is an odd threshold obtained by a large number of experiments, if there are $(T+n) / 2$ similarities in the sequence to be tested, then consider that $\left\{b_{n}\right\}$ is a synchronous signal. 


\section{WATERMARK EMBEDDING ALGORITHM}

\section{A. Pretreatment of the watermark}

In order to improve the robustness of digital watermarking algorithm, making the added watermark can resist signal processing attacks such as shear and resampling methods, and in order to achieve the purpose of security and confidentiality in the meantime, Arnold transform is used in this paper, use the scrambling transform on the binary image $\mathrm{W}$, in which the number of transformation is use as the key. In order to embed the two-dimensional watermark in the one-dimensional digital audio signal, reduction of the dimensions must be done. In addition, to further improve the resistance abilities against simultaneously attack such as shear, translation, etc. An synchronous sequence required to be added in the embedded sequence. The m-sequence is used as a synchronous signal here. Thus, the structure of the final embedded audio signal sequence $V 1$ is shown in Fig. 2.

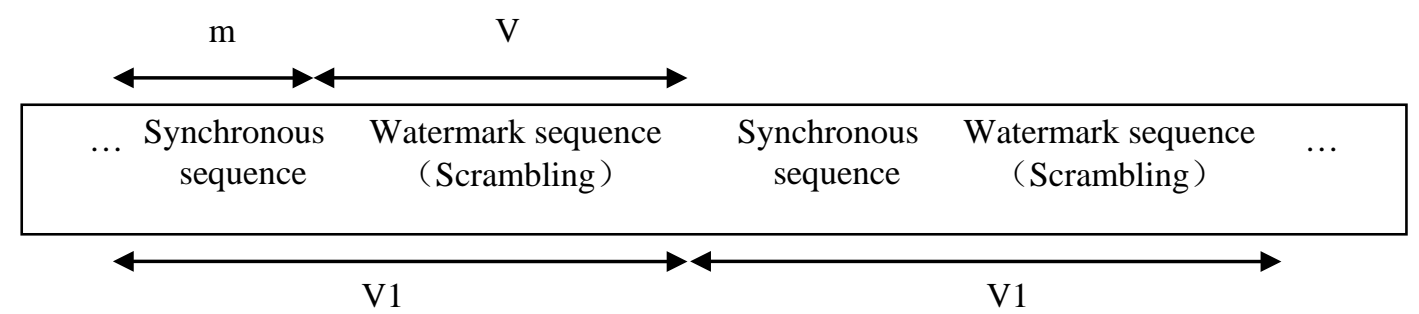

Figure 2. Composition structure of watermark sequence to be embedded

\section{B. Audio signal segments}

Suppose that A represents the original digital audio signal contains $L$ samples of data, It can be expressed as

$$
A=\{a(l), 0 \leq l<L\}
$$

Which $a(l) \in\left\{0,1,2, \ldots,\left(2^{p}-1\right)\right\}$ is the magnitude value of the $l$ th data, ${ }^{p}$ is the bit numbers used for each data. Separate the original audio signal into two parts, the watermark embedded relevant part $A_{e}$ and the watermark embedded irrelevant part $A_{r}$, that is $\quad A=A_{e}+A_{r}$

Assuming the number of data for each audio segment is $L$, the total number of audio data is $N$, embed a pixel value in each data segment, in order to embed all $M 1 \times M 2$ watermark pixels, the number of original audio data that must be met:

$N \geq M 1 \times M 2 \times L$

Divided the audio $A_{e}$ used to embed watermarks into $M 1 \times M 2$ segments

$$
A_{e}=\left\{A_{e}(k), 0 \leq k<(M 1 \times M 2)\right\}
$$

C. Wavelet decomposition of each audio segment

Select the appropriate wavelet base to do discrete wavelet transform on watermark embedded related audio $A_{c}$, Daubechies-4 wavelet base is chosen in this paper, and 3-layer wavelet decomposition is used on related audio, that is

$$
D=D W T\left(A_{e}\right)=C D_{1} \oplus C D_{2} \oplus C D_{3} \oplus C A_{3}
$$

D. Watermark embedding after the low frequency coefficients mean quantization

Consider that most of the audio signal energy concentrated in the low frequency components, with good stability, resistance to noise and other signal processing abilities. To ensure the robustness of the watermark, select $\mathrm{CA}_{3}$ as the region of watermark embedding, its coefficient is denoted by $\left.X=\left\{a_{i} \mid a_{i} \in C A_{3}\right), i=0, \ldots, M-1\right\}, M$ is the sum of the coefficients of the sub-band $C A_{3}$,then the watermark embedding is completed.

E. Obtain the audio signal with watermark by using the inverse wavelet transform

Replace the original ${ }^{C A_{3}}$ with low frequency coefficient ${ }^{C A_{3}^{\prime}}$ obtained by Watermark embedding, the detail component ${ }^{C D_{i}(i=1,2,3)}$ maintains the same, then we have wavelet transformation territory expression after the embedding

$$
A_{e}^{\prime}=I D W T\left(C D_{1} \oplus C D_{2} \oplus C D_{3} \oplus C A_{3}^{\prime}\right)
$$


Finally carry on wavelet reconstruction, replace the original audio signal $A_{e}$ with the audio signal $A_{e}^{\prime}$ with watermark, irrelevant part $A_{r}$ remains the same, then we have the audio signal $A^{\prime}$ with watermark.

$$
A^{\prime}=A_{e}^{\prime}+A_{r}
$$

\section{WATERMARK EXTRACTION ALGORITHM}

A. audio signal segmentation

Separate the audio signal to be tested $A_{s}$ into two parts, the watermark embedded relevant part $A_{s e}$ and the watermark embedded irrelevant part $A_{s r}$, that is

$$
A_{s}=A_{s e}+A_{s r}
$$

Which $A_{s e}=\left\{A_{s e}(k), 0 \leq k<M 1 \times M 2\right\}, A_{s e}(k)$ expresses the $k$ th data segment.

B. Segmentation wavelet transformation

Choose the same wavelet base with the embedded watermark-- Daubechies- 4 wavelet base to do 3-layer wavelet transform on watermark embedded relevant part $A_{\text {se }}$, choose approximate component coefficient after the transformation to do the next step processing, the result of wavelet transformation is:

$$
D_{s}=D W T\left(A_{s e}\right)=C D_{s 1} \oplus C D_{s 2} \oplus C D_{s 3} \oplus C A_{s 3}
$$

C. Watermark extraction

Select the low frequency component ${ }^{C A_{s 3}}$, Use the same measure during embedding to divide coefficient set composed by ${ }^{C A_{3}}$ into array, extract average value of a row. Suppose the average value is $\overline{Y^{\prime}(l)}$, Extract watermark sequence $\left\{w^{\prime}(l)\right\}$ from $\bar{Y}^{\prime}(l)$, that is

$$
w^{\prime}(j)=\left\lfloor\overline{Y^{\prime}}(l) / Q_{1}+1 / 2\right\rfloor \% 2 \quad l=0, \ldots, L-1
$$

\section{Dimension rising and counter scrambling}

A chaotic image $w(i, j)$ is obtained after Dimension rising on the extracted watermark bit $V^{\prime}(l)$, then we can obtain the watermark image by doing inverse Arnold transform according to the key on the chaotic picture.

$$
W^{\prime}=\left\{w^{\prime}(i, j), 1 \leq i \leq M 1,1 \leq j \leq M 2\right\}
$$

\section{WATERMARK EXTRACTION ALGORITHM}

In the experiment, choose a section of music which broadcast time is a 21 second, quantification precision is 16 , the sampling rate is $44100 \mathrm{~Hz}$ and sampling points are 911757as the original signal, its oscillograph is shown in Fig.3. The m-sequence which synchronized signal cycle is 63 and the quantification length of stride is 0.2 cannot notice the differences after inserting watermark in the audio data, the audio frequency oscillograph of embedded watermark is shown in Fig.4.

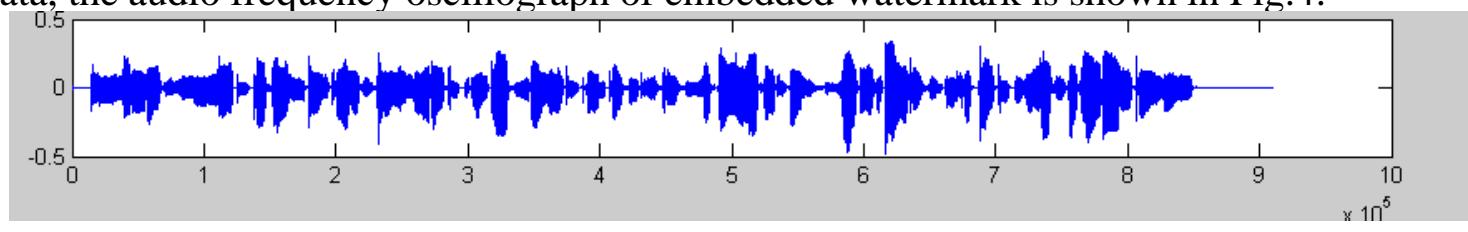

Figure 3. Oscillograph of original audio signal

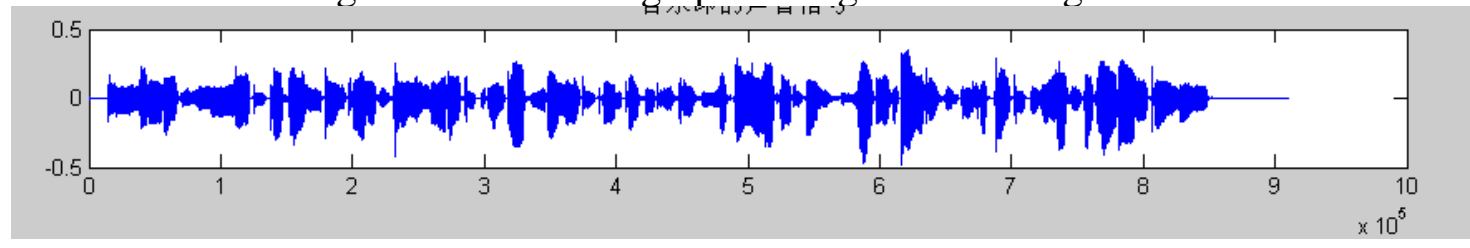

Figure 4. Audio signal with embedded watermark 


\section{CONCLUSION}

In this paper, we proposed a mean quantization based self-synchronous digital audio watermarking method in wavelet domain. In this method, each watermark bit is embedded a group of average wavelet coefficients by quantizing, and the watermark is extracted without the original audio signal. Experimental results show that the mean quantization watermarking system has been enhanced relative to the overall performance of the general quantization based watermarking system; that's to say, without introducing perceptual noise, the watermark robustness of the system have been well improved.

This algorithm has the following characteristics: 1) The algorithm is a blind detection algorithm to detect without the original audio signal; 2)Bringing the concept of coefficient mean quantization, this algorithm can embed watermark after the wavelet decomposition of audio signals and coefficients mean quantization on low frequency; 3) It also bring the concept of embedded synchroniztion signal to determine the position of watermark for resisting the shift, cutting and other attacks.

\section{References}

[1] NIU Xin-xin . YANG Yi-xian. A New Algorithm for Digital Watermarking Based on the Wavelet Transform[J]. Chinese Journal of Computers. 2000, 23( 1)21-27.

[2] Sun Rui.Sun Hong.Yao Tian-ren. Digital audio watermarking algorithm based on quantization in wavelet domain[J]. Journal of Huazhong University of Science and Technology. 2000, 23( 1)21-27.

[3] CHEN Jin-er. WANG Rang-ding. XU Ji. Mean Quantization Audio Watermarking Algorithm Based on Wavelet Transform[J]. Application Research of Computers. 2006, (4):107-110.

[4] ZHOU Ke-lan. ZHANG Yu-hua. Audio Watermarking Based on Psychoacoustic Model and the Wavlet Transform[J]. Microelectronics \& Computer. 2005, 22(3): 185-194.

[5] SHI Hua-ji. ZHOU Xin-fa. LI Xing-yi. DING Qiu-lin. Algorithm of Blind Synchronous Digital Audio Watermarking[J]. Journal of Jilin University(Information Science Edition). 2006,21(1),56-61. 